\title{
MESOSCOPIC SIMULATION OF INCOMPRESSIBLE FLUID FLOW IN POROUS MEDIA
}

\author{
M. Y. Gokhale ${ }^{1}$, Ignatius Fernandes ${ }^{2}$ \\ ${ }^{1}$ Maharashtra Institute of Technology, Pune, Maharashtra, India \\ ${ }^{2}$ Department of Mathematics, University of Pune, Maharashtra, India
}

\begin{abstract}
Lattice Boltzmann method is used to simulate cavity driven fluid flow in porous media. A square cavity is considered with the top lid moving with uniform velocity and other sides kept stationary. Simulation is carried out for values of Darcy number ranging from $10^{-6}$ to $10^{-2}$ at Reynolds number 10 and 100. Influence of Darcy number and Reynolds number is investigated on velocity profiles and the streamline plots. Half-way bounce back boundary conditions are employed in the numerical simulation. The numerical code is first verified with the results available in the literature and then used to simulate the Newtonian fluid flow in porous media. The Darcy number and the Reynolds number were observed to have great influence on the flow properties and the location of the primary vortex. Simulation was carried out for a 100×100 mesh grid and a fine agreement is established theories in incompressible fluid flow.
\end{abstract}

Keywords: Lattice Boltzmann method, incompressible flow, porous media

\section{INTRODUCTION}

Fluid flow through porous media has been an area of great interest over many decades considering its applications in areas such as petroleum extraction, enhanced oil recovery and groundwater flow. Though significant research is available in this area, there is large number of problems which needs further attention. Simulation of transport phenomena in porous media is followed by the complexity in imposing the boundary conditions in the numerical methods, which arises since fluid flow simulation has evolved around solving Navier-Stokes equation. With a revolution in technology, which has increasingly helped researchers to reduce the computational and analytical time, these problems can now be addressed easily. Lattice Boltzmann method (LBM), which evolves from Lattice Gas Automata, has attracted great attention for its ability to simulate fluid flow without having to solve the continuity equations. The simplicity in considering the boundary conditions has been the major advantage of LBM, which is also verified by many researchers as available in the literature.

A large number of researchers have contributed in this area. Gou and Zhao [1] proposed a lattice Boltzmann model for isothermal incompressible flow in porous media which was used to simulate generalized two-dimensional Poiseuille flow, Couette flow, and lid-driven cavity flow. Shi et al [2] simulated mixed convection in a driven cavity packed with porous medium using lattice Boltzmann method. They also proposed a lattice Boltzmann method for incompressible flow in porous media and thermal lattice Boltzmann method for solving the energy equation based on generalized volume-averaged flow model. Mehrizi et al [3] simulated forced convection in vented cavity filled by porous medium with obstruction using LBM. Begum and Basit [4] validated
LBM for different flows and transition process by implementing it on plane Poiseuille flow, planar Couette flow and lid driven cavity flow. A new partial bounce back algorithm for lattice Boltzmann method for fluid flow through heterogeneous media was proposed by Walsh et al [9] which employed a probabilistic meso-scale model that varied individual lattice node properties to reflect a material's local permeability. In another paper Grucelski [10] applied single relaxation time variant of the LatticeBoltzmann Method to the two-dimensional simulations of viscous fluids, which they applied to a geometry consisting of an obstacle and geometry of system of obstacles making up a simple, computer-created porous medium. The main parameters in simulation of fluid flow in porous media are Reynolds number (Re), Darcy number (Da), porosity, permeability and aspect ratio. Though a significant research is carried out for larger value of $\mathrm{Da}$, attention is required at low values of $\mathrm{Da}$, since the major of the natural phenomena such as flow in oil sand and soil occurs at low Da and thus at low permeability values. This paper investigated fluid flow in porous media at low $\mathrm{Da}$ and different Re values. The study will help understand the flow behavior of fluids in porous media at low permeability, which is the situation found during the fluid flow in oil sands and groundwater flow.

\section{GOVERNING EQUATIONS}

In continuum domain, the incompressible fluid flow in porous media is governed by [1]

$\nabla . u=0$

$$
\frac{\partial u}{\partial t}+(u . \nabla)\left(\frac{u}{\varepsilon}\right)=-\frac{1}{\rho} \nabla(\varepsilon p)+v_{e} \nabla^{2} u+F
$$


Where $\rho$ is the fluid density, $\varepsilon$ is the porosity of the medium, $\mathrm{u}$ and $p$ the velocity and pressure, respectively, and $v_{e}$ an effective parameter. $F$ represents the total body force due to presence of a porous medium and other external force fields, and is given by [3]

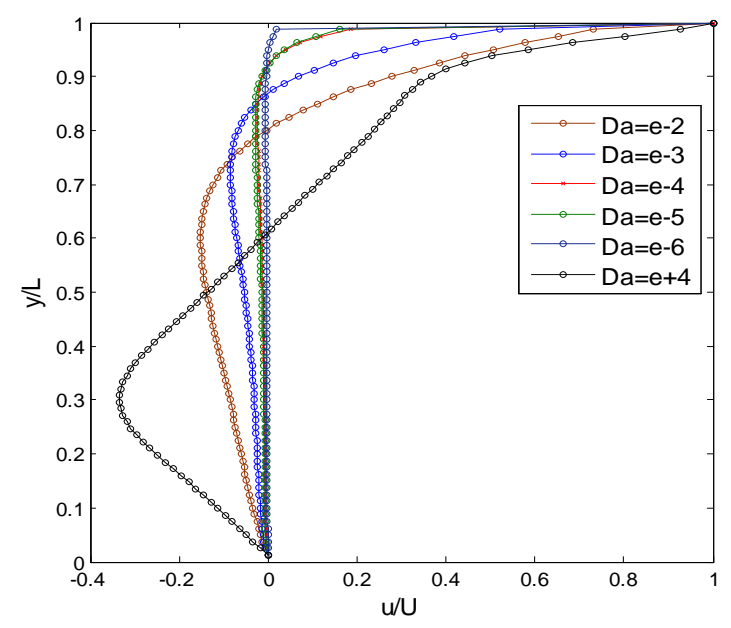

(a)

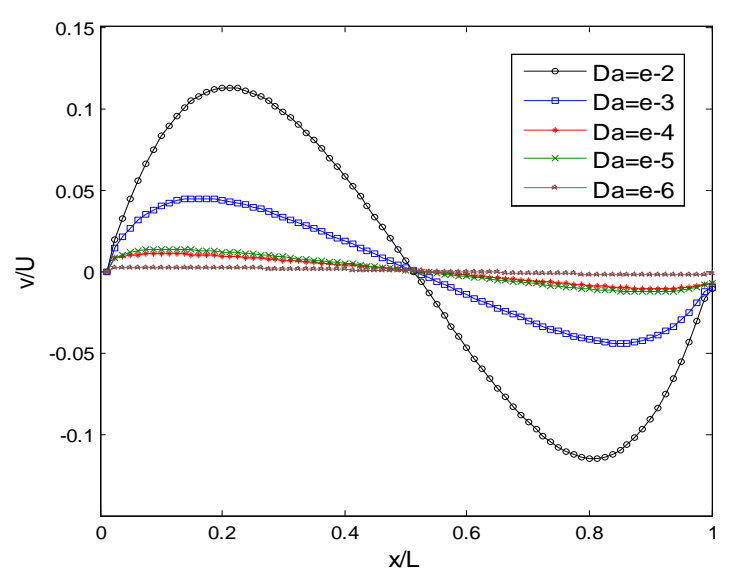

(b)

Fig.1. Velociy profiles for various values of $\mathrm{Da}$ at $\mathrm{Re}=10$. (a) u-velocity profiles (b) v-velocity profiles.

$$
F=-\frac{\varepsilon v}{K} u-\frac{\varepsilon F}{\sqrt{150 \varepsilon K}}|u| u
$$

Where $v$ is the kinematic viscosity and $K$ is the permeability

The permeability is related to Darcy number and the characteristic length $L$ by

$$
K=D a L^{2}
$$

\section{NUMERICAL METHOD}

\subsection{Lattice Boltzmann Method}

The lattice Boltzmann method can be used to model hydrodynamic or mass transport phenomena by describing the particle distribution function $f_{i}(x, t)$ giving the probability that a fluid particle with velocity $e_{i}$ enters the lattice site $x$ at a time $t$.

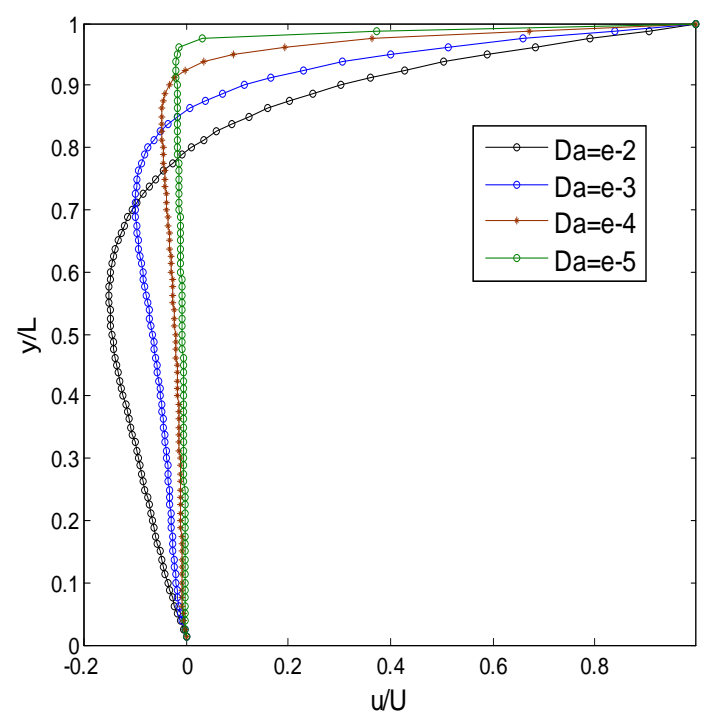

(a)

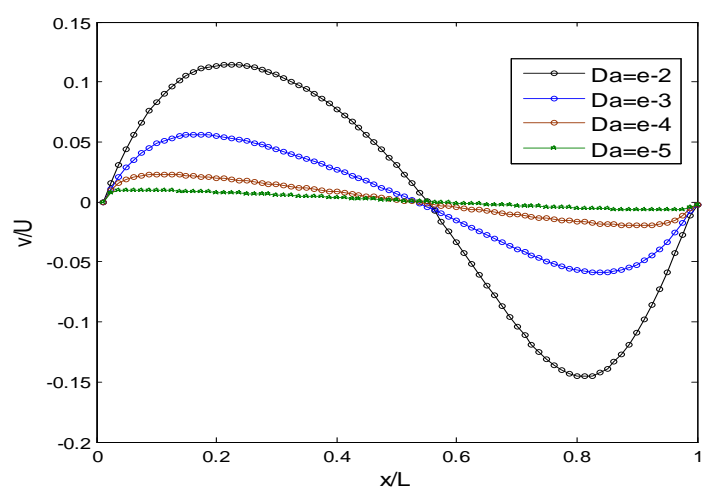

(b)

Fig.2 Velocity profiles for various values of $\mathrm{Da}$ at $\mathrm{Re}=100$. (a) u-velocity profiles (b) v-velocity profiles.

The subscript $i$ represents the number of lattice links and $i=0$ corresponds to the particle at rest residing at the center. The evolution of the particle distribution function on the lattice resulting from the collision processes and the particle propagation is governed by the general form of the discrete Boltzmann equation with external force $[2,5,6]$

$$
f_{i}(x+, t+\Delta t)-f_{i}(x, t)=\Delta t \frac{f_{i}^{e q}(x, t)-f_{i}(x, t)}{\tau}+\Delta t F_{i}
$$




$$
f_{i}\left(x+e_{i} \Delta t, t+\Delta t\right)=f(x, t+\Delta t)
$$

Equation (4) and (5) are the collision and streaming steps, respectively.

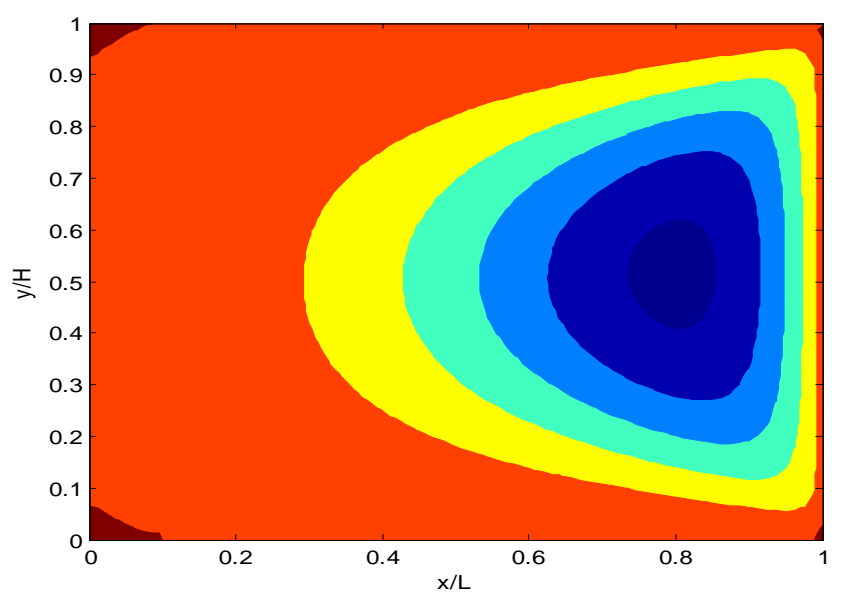

(a)

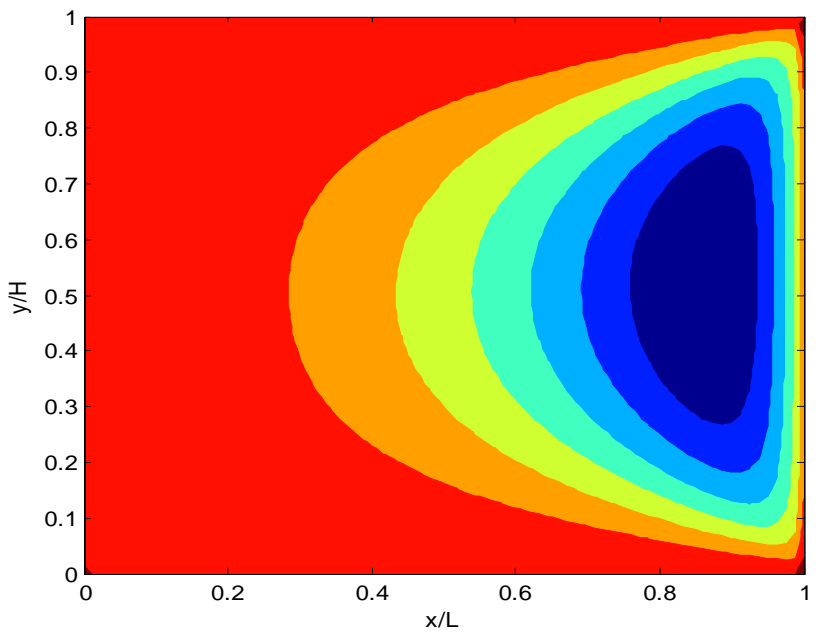

(b)

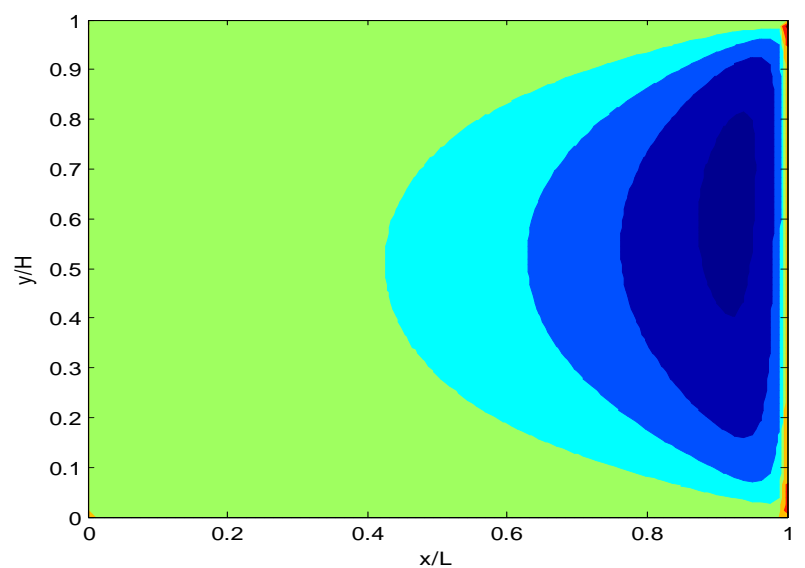

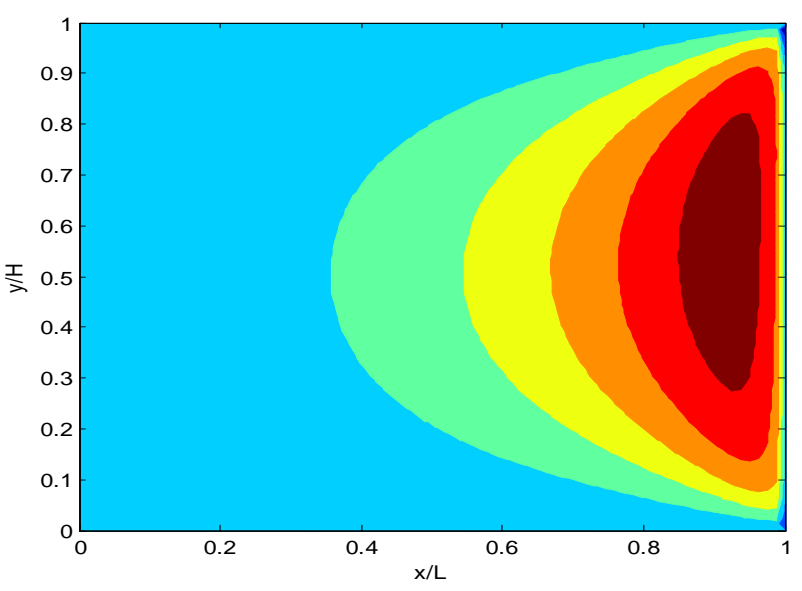

(d)

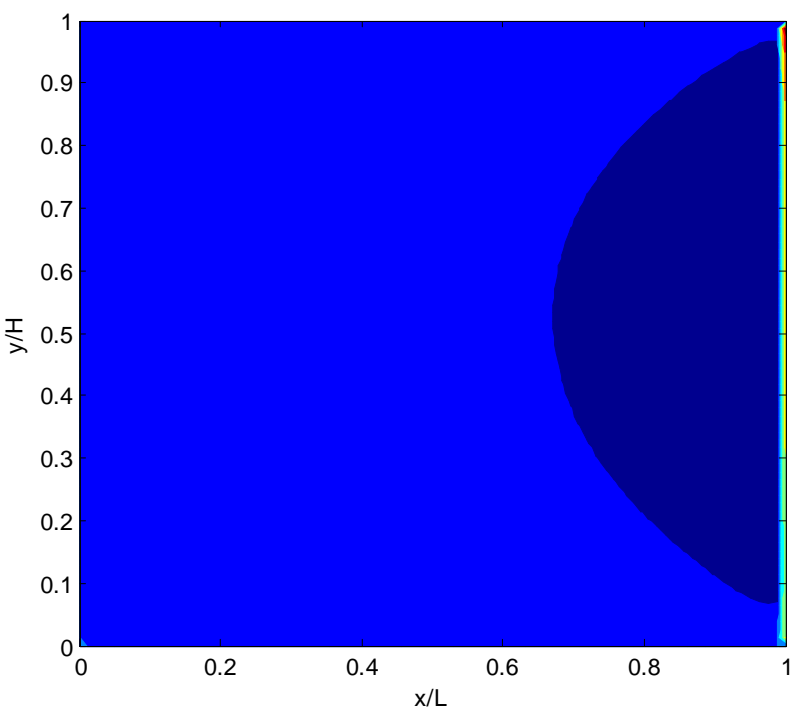

(e)

Fig 3. Streamline plots for different values of $\mathrm{Da}$ at $\mathrm{Re}=10$. (a) $\mathrm{Da}=10^{-2}$ (b) $\mathrm{Da}=10^{-3}$ (c) $\mathrm{Da}=10^{-4}$ (d) $\mathrm{Da}=10^{-5}$ (e) $\mathrm{Da}=10^{-}$

$f_{i}^{e q}(x, t)$ is the equilibrium distribution function given by [3]

$$
f_{i}^{e q}(x, t)=\omega_{i} \rho\left[1+\frac{e_{i} \cdot u}{e_{i}{ }^{2}}+\frac{1}{2} \frac{\left(e_{i} \cdot u\right)^{2}}{\varepsilon e_{i}{ }^{4}}-\frac{1}{2} \frac{u^{2}}{\varepsilon e_{i}{ }^{2}}\right]
$$

(6)

The forcing term $F_{i}$ is given by $[1,7,8]$

$$
F_{i}=\omega_{k} \rho\left(1-\frac{1}{2 \tau_{v}}\right)\left[\frac{e_{i} \cdot F}{e_{i}{ }^{2}}+\frac{\left(u F: e_{i} e_{i}\right)}{\varepsilon e_{i}{ }^{4}}-\frac{u . F}{\varepsilon e_{i}{ }^{2}}\right]
$$

(c) 
The forcing term $F_{i}$ defines the fluid velocity as

$$
u=\sum_{i} \frac{e_{i} F_{i}}{\rho}+\frac{\Delta t}{2} F
$$

Equation (8) relates $F$ to $u$ which is nonlinear for the velocity. To overcome this, Gou and Zhao [6] presented a temporal velocity $V$ given by

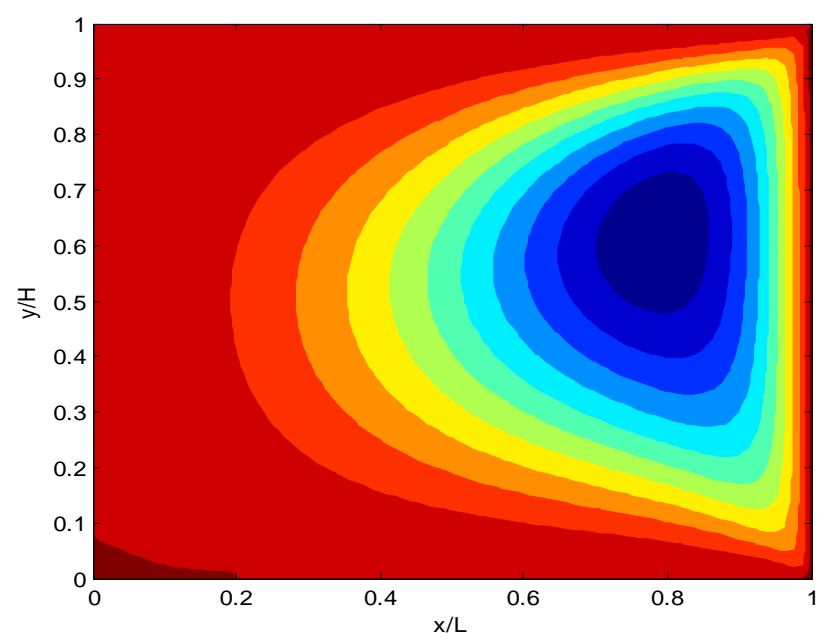

(a)

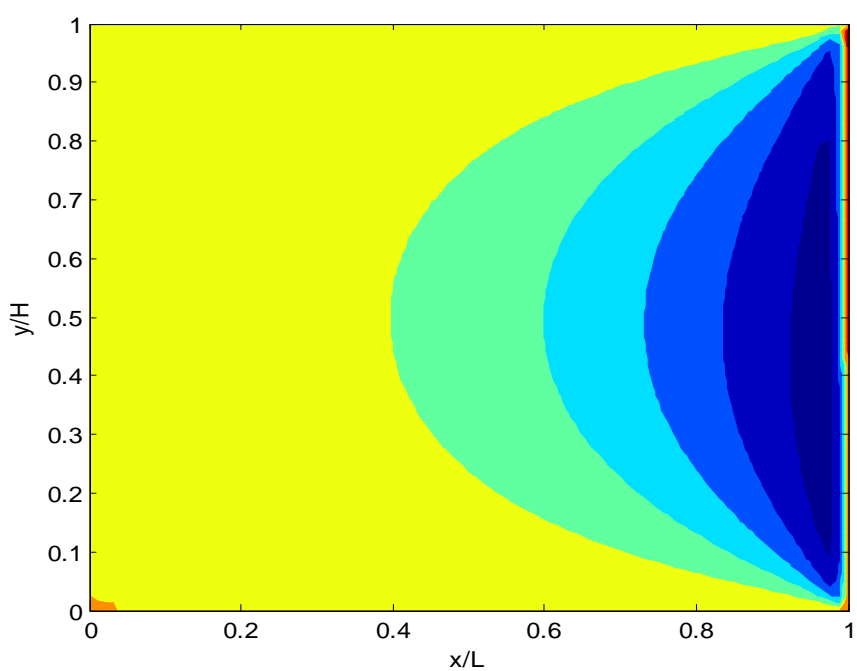

(b)

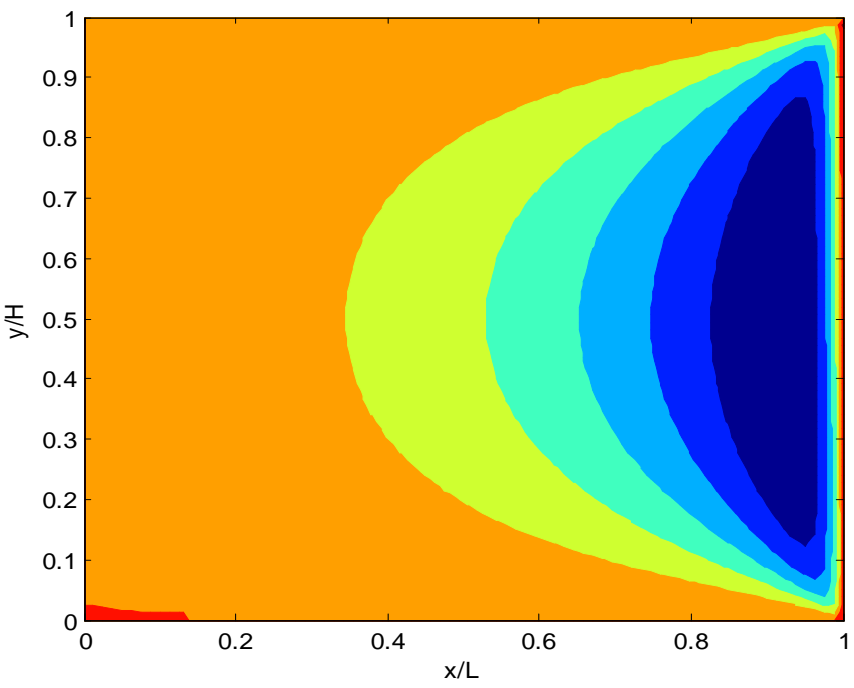

(c)

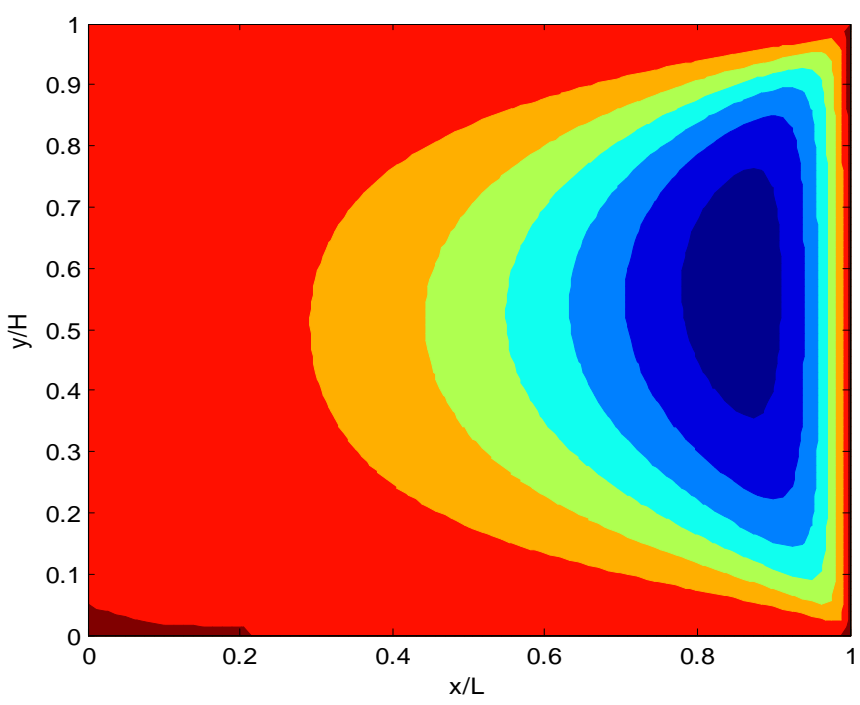

(d)

Fig 4. Streamline plots for different values of $\mathrm{Da}$ at $\mathrm{Re}=10$. (a) $\mathrm{Da}=10^{-2}$ (b) $\mathrm{Da}=10^{-3}$ (c) $\mathrm{Da}=10^{-4}$ (d) $\mathrm{Da}=10^{-5}$.

$$
u=\frac{V}{c_{0}+\sqrt{c_{0}^{2}+c_{1}|V|}}, V=\sum_{i} \frac{e_{i} f_{i}}{\rho}
$$

(9)

The two parameters $c_{0}$ and $c_{1}$ are given by

$$
c_{0}=\frac{1}{2}\left(1+\varepsilon \frac{\Delta t v}{2 K}\right), c_{1}=\varepsilon \frac{\Delta t}{2} \frac{1.75}{\sqrt{150 \varepsilon^{3} K}}
$$

\subsection{Numerical Implementation}

Fluid flows through the cavity with the top lid moving from left to right with a uniform velocity of $U=u_{0}=0.1$. The velocities at all other nodes are assumed to be zero considering these sides to be stationary. The height of the 
cavity is $H$ and the length of the horizontal sides is $L$. A uniform fluid density $\rho=1.0$ is imposed initially. The porosity $\varepsilon$ is set to 0.1 and the relaxation parameter $\tau$ is assumed to be 0.8. Half-way bounce back boundary conditions are used in the numerical simulation. The solution procedure of the above LBM at each time step comprises of streaming and collision steps, application of boundary conditions, calculation of particle distribution function followed by calculation of macroscopic variables. The numeric simulation is carried out for values of $\mathrm{Da}$ between $10^{-6}$ and $10^{-2}$ and at Re 10 and 100. A MATLAB code is developed for a $100 \times 100$ mesh grid.

\section{RESULTS AND DISCUSSIONS}

Simulation was carried to investigate the influence of Darcy number and Reynolds number on the velocity profiles and the streamline plots for a range of values from $10^{-6}$ and $10^{-2}$ for $\mathrm{Da}$ at $\mathrm{Re} 10$ and 100. First, the MATLAB code was verified by comparing the results for $\mathrm{Da}=10^{4}$ at $\mathrm{Re}=400$ with [1]. The result is shown in Fig 1 and shows fine agreement with established results. Fig 1a presents u-velocity profiles at the center of the cavity for various values of $\mathrm{Da}$ for $\mathrm{Re}=10$. The lower values of $\mathrm{Da}$ are related to lower values of permeability. This influences the flow of the fluid, as indicated by the velocity profiles. The u-velocity values tend to decrease in magnitude as Da decreases, with velocity becoming almost zero for low Da. The basic trend in $\mathrm{u}$ velocity profiles remains the same, as observed from the figure, the u-velocity starts at zero (on the boundary), decreasing to attain minimum negative value and then again increases to become zero at the center of the cavity. The uvelocity then increases to attain the maximum positive value. The nature of the profiles is also same for $\mathrm{Re}=100$, except that the magnitude of the u-velocity increases, as shown in Fig 2a. This is naturally related to the rheological behavior of the fluid. The maximum u-velocity is observed at the upper side of the cavity which is due to the effect of moving lid. The v-velocity profiles for $\mathrm{Re}=10$ are presented in Fig1b. As observed, the v-velocity starts from zero, increasing to reach the highest positive value and then decreases to become zero at the center of the cavity. The vvelocity then decreases to reach the minimum negative value, and then increasing to reach zero at the other side of the cavity. The maximum v-velocity increases and the minimum v-velocity decreases with an increase in the values of Re, as observed in Fig 2b. Fig 3-4 presents the streamline plots for $\mathrm{Re}=10$ and $\mathrm{Re}=100$. For $\mathrm{Re}=10$, the streamlines are observed towards right of the cavity, as seen from Fig 3. The primary circulation is concentrated towards the right vertical side of the cavity, with streamlines parallel to the side, indicating a weak circulation in the region. This observation can naturally be related to the value of Da being smaller. With an increase in the values of Re, the strength of the primary circulation is observed to increase, and covers bigger part of the cavity, as observed in Fig 4. For lower values of $\mathrm{Da}$, the streamlines are very weak, the strength of which increases with increase in Re. The primary vortex shifts slightly towards the center of the cavity.

\section{CONCLUSIONS}

The paper presented simulation of incompressible fluid flow in a cavity driven containing porous media. Simulation was carried out for low values of Darcy number ranging from $10^{-}$ ${ }^{6}$ to $10^{-2}$ at Reynolds number 10 and 100 . The velocity profiles and the streamline plots were found to be greatly influenced by Darcy number and Reynolds number. For low values of Darcy number, the velocity profiles showed very weak circulation in the cavity because of low permeability. However, the strength of the circulation increased with an increase in Reynolds number from 10 to 100 . Though trend of the velocity profiles remains the same, circulations expands to bigger part of the cavity for Reynolds number 100. Recirculation was also observed for higher values of Darcy number which corresponds to higher values of permeability.

\section{REFERENCES}

[1] Guo Zhaoli, Zhao T. S., Lattice Boltzmann model for incompressible flows through porous media, Physical Review, American Physical Society, E 66, 036304 (2002).

[2] Shi Baochang, Chai Zhenhua, Guo Zhaoli, Lattice Boltzmann Simulation of Mixed Convection in a Driven Cavity Packed with Porous Medium, ICCS 2007, Part I, LNCS 4487, pp. 802-809, (2007).

[3] Mehrizi A. Abouei, Sedighi K., H. Hassanzade Afrouzi, A. Latif Aghili, Lattice Boltzmann Simulation of Forced Convection inVented Cavity Filled by Porous Medium with Obstruction, World Applied Sciences Journal 16 (Special Issue of Applied Math): 31-36, 2012.

[4] Basit M. Abdul, Romana Begum, Lattice Boltzmann method and its applications to fluid flow problems, European Journal of Scientific Research, Vol 22 No.2 (2008) 216-231.

[5] A. Mohamad, Lattice Boltzmann method, SpringerVerlag London Limited 2011.

[6] Shiyi Chen, Gary D. Doolen, Lattice Boltzmann method for fluid flows, Annu. Rev. Fluid Mech. 30 (1998) 329-364.

[7] Seta, T., E. Takegoshi, K. Kitano and K. Okui, Thermal lattice Boltzmann model for incompressible flows through porous media, Journal of Thermal Science and Technology(2006), 1 (2) 90-100.

[8] Peng, Y., C. Shu and Y.T. Chew, A 3D incompressible thermal lattice Boltzmann model and its application to simulate natural convection in a cubic cavity, J. Comput. Phys (2004), 193 (1) 260274. 
[9] Walsh Stuart D. C., Burwinkle Holly, Saar Martin O., A new partial-bounceback lattice-Boltzmann method for fluid flow through heterogeneous media, Computers and Geosciences 35 (2009) 1186-1193.

[10] Grucelski A., Pozorski J., Lattice Boltzmann simulations of flow past a circular cylinder and in simple porous media, Computers \& Fluids 71 (2013) $406-416$. 